Tecnología y

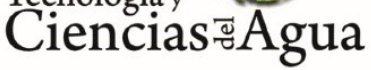

2020, Instituto Mexicano de Tecnología del Agua

Open Access bajo la licencia CC BY-NC-SA 4.0

(https://creativecommons.org/licenses/by-nc-sa/4.0/)

DOI: $10.24850 /$ j-tyca-2020-01-03

Artículos

\title{
Tendencia de la sequía meteorológica en el estado de Durango, México, por el método de Rodionov
}

\section{Trend of meteorological drought in the state of Durango, Mexico, by the Rodionov method}

Janeth Cortez-Villa ${ }^{1}$

Abel Quevedo-Nolasco ${ }^{2}$

Ramón Arteaga-Ramírez ${ }^{3}$

Guillermo Carrillo-Flores ${ }^{4}$

${ }^{1}$ Colegio de Postgraduados, Campus Montecillo, Carretera MéxicoTexcoco km 36.5, Texcoco, Estado de México, México, janeth.cortez.villa@gmail.com, ORCID: 0000-0003-0049-3362

${ }^{2}$ Colegio de Postgraduados, Campus Montecillo, carretera MéxicoTexcoco km 36.5, Texcoco, Estado de México, México, anolasco@colpos.mx, ORCID: 0000-0003-3303-5077 
2020, Instituto Mexicano de Tecnología del Agua

Open Access bajo la licencia CC BY-NC-SA 4.0

(https://creativecommons.org/licenses/by-nc-sa/4.0/)

${ }^{3}$ Universidad Autónoma Chapingo, Carretera México-Texcoco km. 38.5, Texcoco, Estado de México, México, arteagar@correo.chapingo.mx, ORCID: 0000-0001-9459-3588

${ }^{4}$ Colegio de Postgraduados, Campus Montecillo, carretera MéxicoTexcoco km 36.5, Texcoco, Estado de México, México, gflores@colpos.mx

Autor para correspondencia: Abel Quevedo Nolasco, anolasco@colpos.mx

\section{Resumen}

La sequía es el fenómeno natural que afecta a más personas en el mundo, hasta la última consecuencia, la muerte de plantas, animales y seres humanos. Para evaluar los posibles daños que causa, se desarrollaron índices de sequía que facilitan su análisis cuantitativo para realizar una planeación y manejo de los recursos hídricos. Sin embargo, actualmente se observan cambios en el sistema climático global que provocan condiciones de sequía de mayor intensidad, duración y frecuencia. Por lo que se realizó la detección de cambios con el método de Rodionov en los índices de sequía SPEI (Índice de Precipitación y Evapotranspiración Estandarizado) y SPI (Índice de Precipitación Estandarizada) a escalas de 6 y 12 meses en el estado de Durango, 
2020, Instituto Mexicano de Tecnología del Agua

Open Access bajo la licencia CC BY-NC-SA 4.0

(https://creativecommons.org/licenses/by-nc-sa/4.0/)

México. Se analizaron las tendencias de cambios en la media (RSI) y varianza (RSSI) de los índices en 49 estaciones del índice SPI de 6 meses $y$ en 45 del índice SPEI de seis meses. Los valores RSI identificaron el comienzo de los periodos de sequía, la mayoría de los valores máximos de RSI se presentaron en julio de 1992. Las tendencias de la frecuencia de valores RSI, disminuyó durante el periodo 20002004 y aumentó durante 2005-2008. La década de mayores cambios en la media fue 1990-1999. La mayor frecuencia de valores RSSI fue en el periodo 1985-1989, los cambios se mantuvieron constantes durante los últimos 20 años. La década de mayores cambios en la varianza fue de 1980-1989.

Palabras clave: Durango, sequía, SPI, SPEI, Rodionov, cambios de media, cambios de varianza.

\section{Abstract}

Drought is the natural phenomenon that affects the most people in the world, until the last consequence, the death of plants, animals, and humans. To evaluate the possible damages it causes, drought indices were developed that facilitate its quantitative analysis to carry out planning and management of water resources. However, there are currently changes in the global climate system, which cause drought conditions of greater intensity, duration, and frequency. Therefore, 
2020, Instituto Mexicano de Tecnología del Agua

Open Access bajo la licencia CC BY-NC-SA 4.0

(https://creativecommons.org/licenses/by-nc-sa/4.0/)

changes were detected with the Rodionov method in the SPEI (Standardized Precipitation Evapotranspiration Index) and SPI (Standardized Precipitation Index) indices at 6 and 12 month scales in the state of Durango, Mexico. We analyzed the trends of changes in the mean (RSI) and variance (RSSI) of the indices in 49 stations of the SPI index for 6 months and in 45 of the SPEI index for 6 months. The RSI values identified the beginning of the drought periods, most of the maximum RSI values were presented in July 1992. Trends in the frequency of RSI values decreased during the 2000-2004 period and increased during 2005-2008. The decade of greatest changes in the average was 1990-1999. The highest frequency of RSSI values was in the 1985-1989 period, the changes have remained constant during the last 20 years. The decade of greatest change in variance was 19801989.

Keywords: Durango, drought, SPEI, SPI, Rodionov, mean changes, changes of variance.

Recibido: $24 / 11 / 2018$

Aceptado: 27/03/2019 
Tecnología y

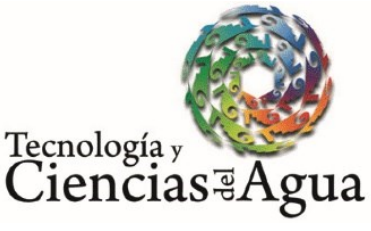

2020, Instituto Mexicano de Tecnología del Agua

Open Access bajo la licencia CC BY-NC-SA 4.0

(https://creativecommons.org/licenses/by-nc-sa/4.0/)

\section{Introducción}

La sequía es un fenómeno climático que ha afectado a la humanidad a través de la historia (Heim, 2002), con mayor impacto en las personas en el planeta (Ortega, 2013). El Instituto Nacional de Ecología y Cambio Climático (INECC) indica que la sequía es de los eventos más costosos para el país (Ibarrarán \& Rodríguez, 2007).

Los impactos de la sequía son muy variados, desde la muerte de plantas y animales, e inclusive la de seres humanos (Cenapred, 2002), efectos sociales en México, con un marcado efecto en el Norte de México (Domínguez, 2016). Una sequía no se puede predecir, pero sí es posible planear y manejar de modo adecuado los recursos hídricos, a fin de mitigar el posible impacto de los daños que pueda causar la sequía (Cancelliere \& Salas, 2010).

Así, es necesario un análisis del fenómeno espacial y temporal, entre las opciones existen índices de sequía, que tienen por objetivo la caracterización de ésta bajo diferentes puntos de vista (Hounam, Burgos, Kalik, Palmer, \& Rodda, 1975).

Los índices de sequía facilitan el análisis cuantitativo sobre la magnitud (con una escala) de la sequía y con ello analizar la distribución 
2020, Instituto Mexicano de Tecnología del Agua

Open Access bajo la licencia CC BY-NC-SA 4.0

(https://creativecommons.org/licenses/by-nc-sa/4.0/)

espacial y temporal del fenómeno, e incluso su frecuencia. Dichos estudios permiten hacer un análisis, evaluación sobre sectores más vulnerables, y contar con información útil para la gestión de riesgos en los grupos afectados (OMM, 2016).

En este marco se deben considerar los cambios en el sistema climático global que se asocian con condiciones de sequía de mayor intensidad, y de mayor duración y frecuencia (Bates, Kundzewicz, Wu, \& Palutikof, 2008). Si bien estas variaciones en el clima son ciertamente naturales, algunos componentes podrían asociarse con efectos antrópicos (Miller, Cayan, \& Barnett, 1994).

En la década de 1970, la interpretación de las variaciones en los registros climáticos derivó en la aceptación del término "cambios de régimen" (Rodionov, 2005a), que son las reorganizaciones rápidas de los ecosistemas de un estado relativamente estable a otro (Rodionov \& Overland, 2005).

Se han desarrollado diversos métodos para detectar cambios de régimen, o discontinuidad, en series de tiempo. Típicamente, estos métodos emplean técnicas estadísticas estándar, como las pruebas de Student o Mann-Kendall, o sus modificaciones (Rodionov, 2004). Un problema común de los métodos existentes para la detección de cambios de régimen es su bajo rendimiento al final de las series temporales. En consecuencia, los cambios en los índices ambientales por lo general se detectan mucho después de su aparición real (Rodionov \& 
2020, Instituto Mexicano de Tecnología del Agua

Open Access bajo la licencia CC BY-NC-SA 4.0

(https://creativecommons.org/licenses/by-nc-sa/4.0/)

Overland, 2005). Como solución a este problema, Rodionov (2004) propone el uso de una técnica de procesamiento de datos secuencial, en la cual se realiza una comprobación para determinar si una nueva observación representa una desviación estadísticamente significativa del valor medio del régimen actual y puede considerarse como un cambio de régimen.

En este trabajo se evalúa la sequía a través del Índice de Precipitación Estandarizada (SPI), y el Índice de Precipitación y Evapotranspiración Estandarizado (SPEI) en el estado de Durango, México (con áreas muy afectadas por la sequía). Se aplicó la prueba de Rodionov para detectar cambios de régimen de media y varianza de los índices de sequía, e identificar si existe tendencia de un incremento o disminución de la ocurrencia de cambios en los últimos años.

\section{Materiales y métodos}

\section{Descripción del área de estudio}


El estado de Durango se ubica en el noroeste de México y tiene una superficie de $123367 \mathrm{~km}^{2}$, cuenta con 39 municipios (INEGI, 2017). Con cuatro provincias fisiográficas (Semarnat, 2009), de mayor a menor extensión están la Provincia Sierra Madre Occidental (porción central y occidental de la entidad); las Llanuras del Norte (en la zona Noreste); la Mesa del Centro (parte centro-oriental), y la Sierra Madre Oriental (Figura 1).

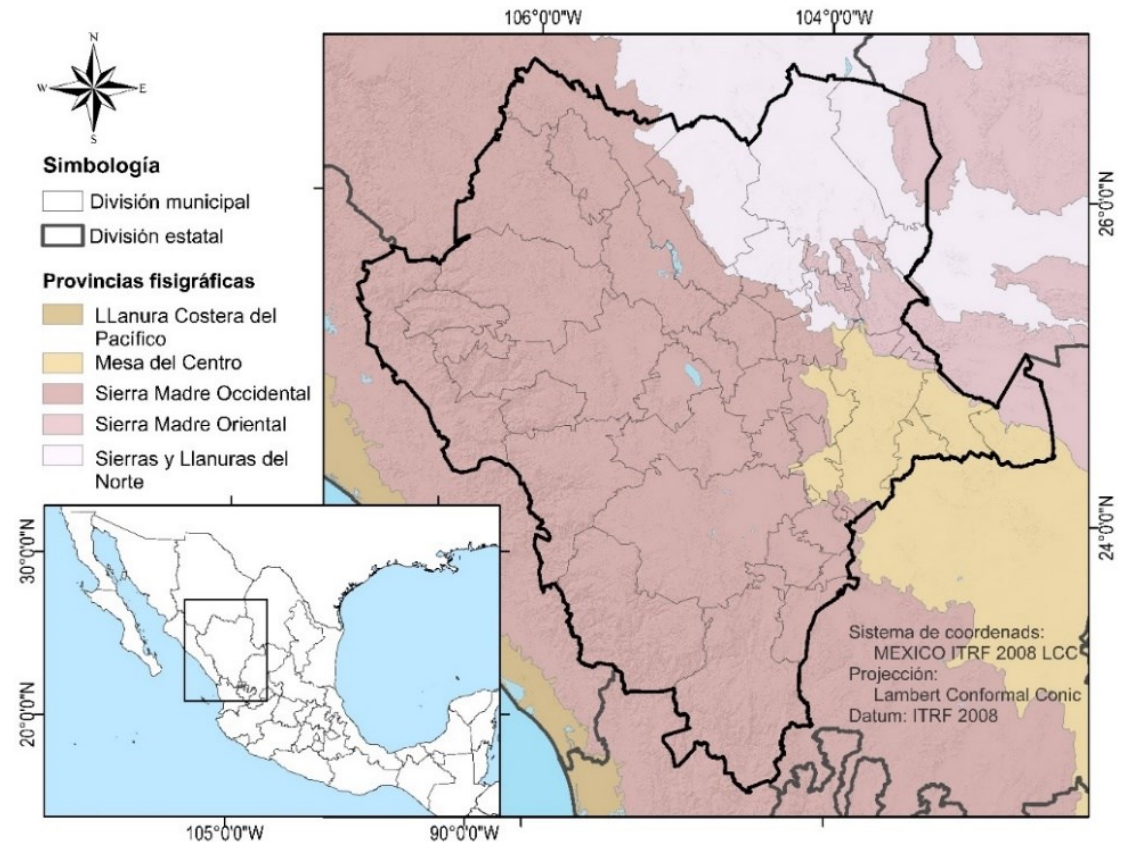

Figura 1. Ubicación del estado de Durango, México, y provincias fisiográficas. 
Ciencias $\widetilde{\mathbb{\Xi}}$ Agua

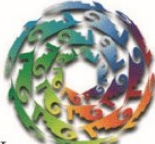

2020, Instituto Mexicano de Tecnología del Agua

Open Access bajo la licencia CC BY-NC-SA 4.0

(https://creativecommons.org/licenses/by-nc-sa/4.0/)

Por su localización geográfica, Durango presenta climas semisecos templados, secos, muy secos, semifríos, semicálidos y cálidos. La distribución de las isoyetas es de suroeste a noroeste en orden decreciente, siendo la mayor de $1200 \mathrm{~mm}$ y la de menor de $200 \mathrm{~mm}$; esta última ubicada en el límite con el estado de Coahuila (Figura 2).

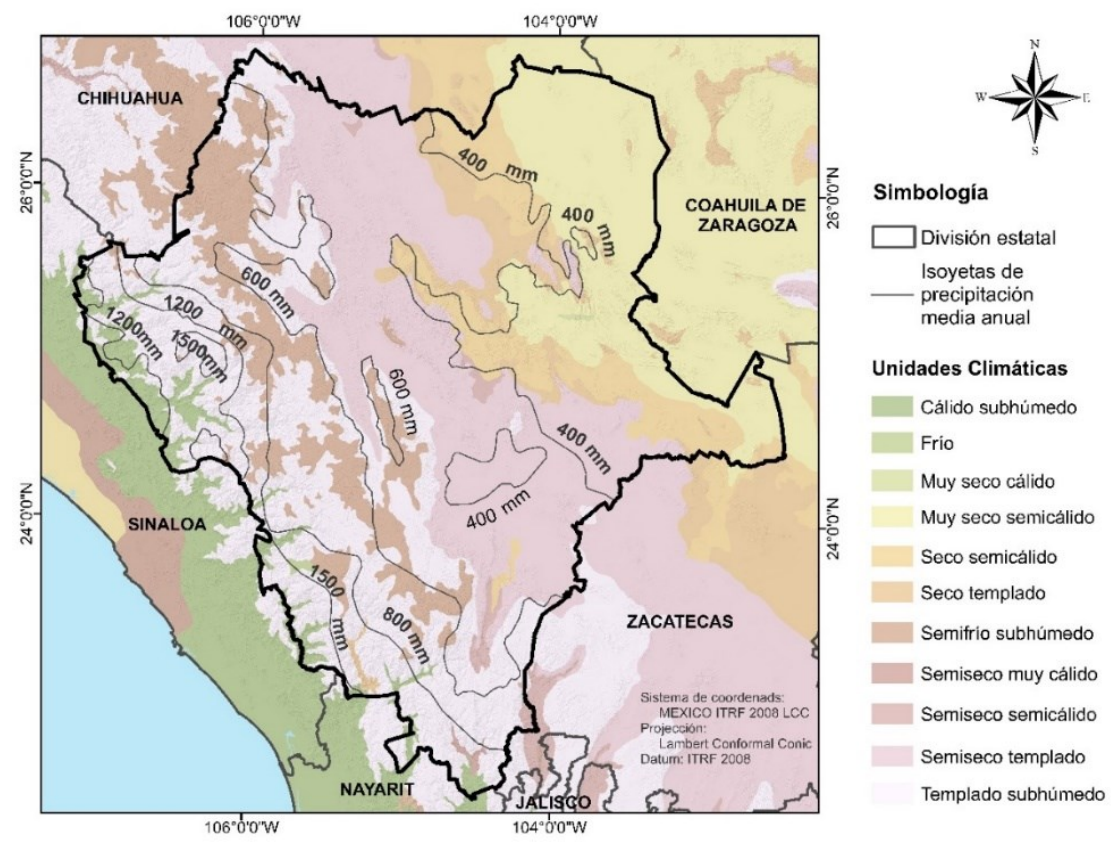

Figura 2. Distribución climática e isoyetas del estado de Durango, México.

En la vertiente del Pacífico, región que corre de noroeste a suroeste, se considera como la zona con mayores precipitaciones (lluvia promedio de $1200 \mathrm{~mm}$ ), que coincide con las zonas de bosque y selva de Durango. En esta parte del estado se encuentra la Sierra Madre 
2020, Instituto Mexicano de Tecnología del Agua

Open Access bajo la licencia CC BY-NC-SA 4.0

(https://creativecommons.org/licenses/by-nc-sa/4.0/)

Occidental, la cual, al recibir la humedad del Pacífico, y al condesar por enfriamiento al ascender, conforma los eventos más importantes de lluvia.

Sólo una pequeña parte de la humedad del Pacífico logra traspasar la barrera orográfica hacia la vertiente interior de Durango, en la zona de pastizales, matorral y agricultura. Con una precipitación media de $600 \mathrm{~mm}$ anuales, y cada vez menores, conforme se adentra a la parte oriental, donde la precipitación media es de $200 \mathrm{~mm}$ al año, se presentan los climas semiseco, seco y muy seco (Semarnat, 2009).

\section{Información meteorológica}

La información meteorológica de las variables de temperatura máxima y mínima, y precipitación a escala diaria de las estaciones meteorológicas que se ubican dentro del área de estudio, se recuperó de las bases de datos del Servicio Meteorológico Nacional (SMN). Se descargó la información mensual por estación del sitio web smn.cna.gob.mx/es/climatologia/informacion-climatologica. 
Tecnología y

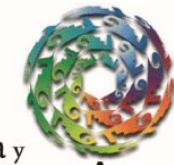

Ciencias
2020, Instituto Mexicano de Tecnología del Agua

Open Access bajo la licencia CC BY-NC-SA 4.0

(https://creativecommons.org/licenses/by-nc-sa/4.0/)

Se eligió como periodo de estudio los años de 1979 a 2008, ya que incluye un mayor número de estaciones con registros (con al menos un $90 \%$ y algunas con $75 \%$ de registros en zonas con poca información) en el periodo de estudio. Con un total de 61 estaciones meteorológicas del SMN, de las cuales 52 pertenecen al estado de Durango, tres a Coahuila, tres a Sinaloa, dos al estado de Zacatecas y una a Chihuahua (Figura 3).

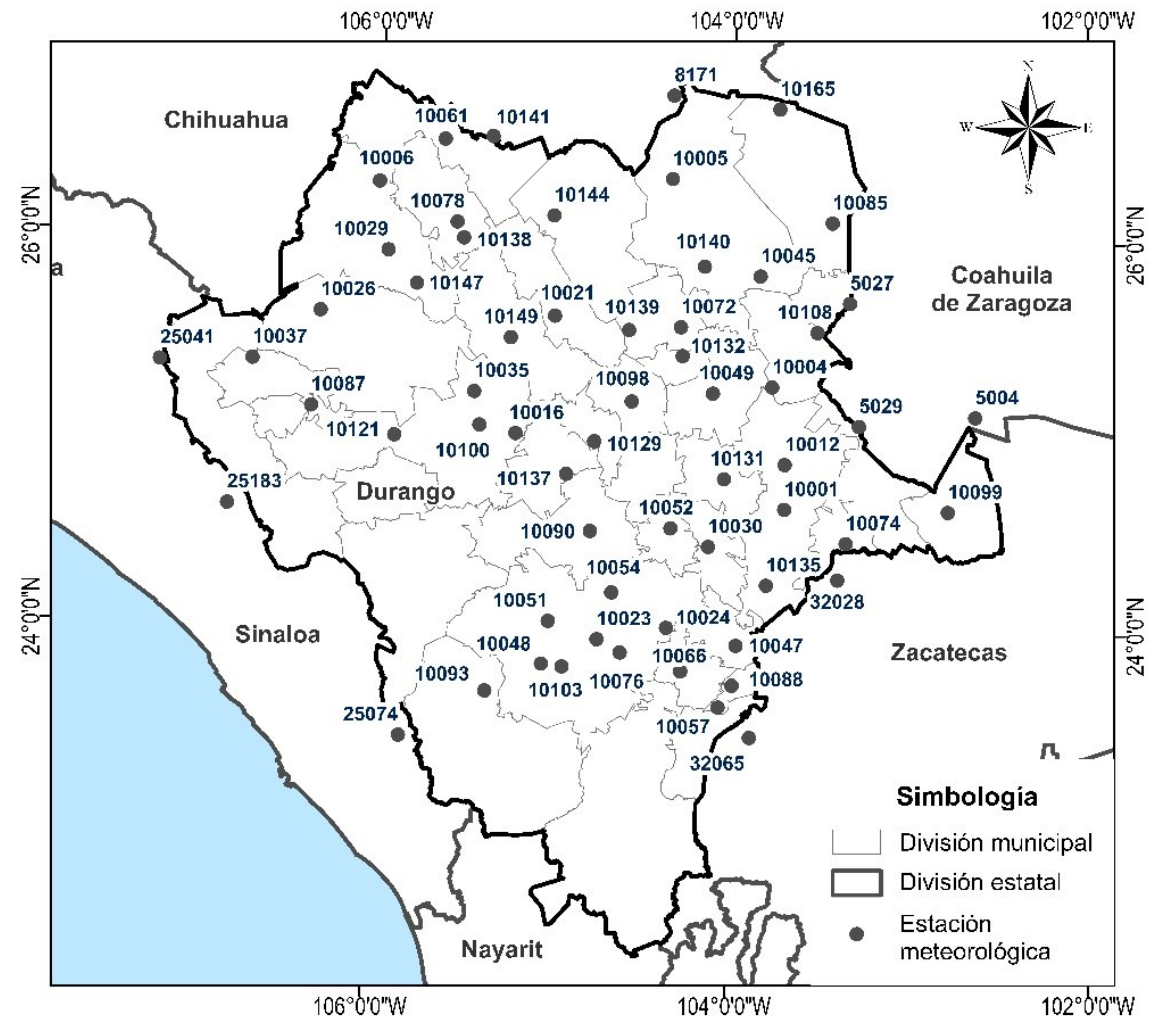

Figura 3. Estaciones meteorológicas SMN seleccionadas. 
A la información meteorológica se le aplicó un control de calidad de datos, que consistió en identificar valores no razonables de acuerdo con los siguientes criterios: (1) cantidad de precipitación diaria menor que cero, y (2) temperatura máxima diaria menor que la temperatura mínima diaria. Se eliminaron los valores no razonables y se trataron como datos faltantes.

Los datos faltantes se completaron de dos formas. El primero, con el método del US National Weather Service (NWS) —conocido también como el cuadrado de la distancia inversa- es un método sencillo de utilizar, pero se debe contar con información de estaciones cercanas (Campos, 1998); puede ser aplicado a datos diarios, mensuales o anuales. Trabajos recientes indican que es uno de los mejores métodos para estimar datos faltantes en México (Gallegos, Arteaga, Vázquez, \& Juárez, 2016; Gamboa, 2015). El segundo, es el modelo Climate Forecast System Reanalysis (CFSR), que desarrolló el National Center for Enviromental Prediction (NCEP) y el National Center for Atmospheric Research (NCAR) de EUA. Se trata de un modelo que representa la interacción global entre los océanos, la tierra y la atmósfera (Saha et al., 2010), se cuenta con un periodo de 35 años (1979-2014) de datos diarios para las variables de temperatura, precipitación, viento, humedad relativa y energía solar; la resolución del CFSR es de unos 38 km (Saha et al., 2014). 
El método NWS estima el valor faltante en la estación de acuerdo con la Ecuación (1):

$$
P x=\frac{\sum P i * W i}{\sum W i}
$$

Donde $P i$ es la precipitación observada en la estación auxiliar $i ; i=$ $1,2,3 \ldots n$, para la fecha faltante; $n$ es el número de estaciones auxiliares (mínimo $n=2$ ).

$W i=\frac{1}{D i^{2}}$

Di es la distancia entre cada estación auxiliar y la estación incompleta, en kilómetros.

Para cada estación se ubicaron las más cercanas como auxiliares y se calculó la distancia entre ellas. Cuando fue posible, se ubicaron las cuatro estaciones auxiliares más cercanas, una sobre cada cuadrante de los ejes coordenados norte-sur y este-oeste (Campos, 1998).

Los datos del modelo CFSR se obtuvieron del sitio web globalweather.tamu.edu. Se realizó la descarga de valores de la malla 
Tecnología y

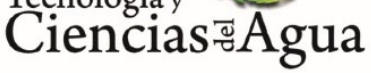

2020, Instituto Mexicano de Tecnología del Agua

Open Access bajo la licencia CC BY-NC-SA 4.0

(https://creativecommons.org/licenses/by-nc-sa/4.0/)

de puntos para el estado de Durango. Para completar los datos faltantes, se eligió el punto de la malla más cercano a cada estación meteorológica (Figura 4).

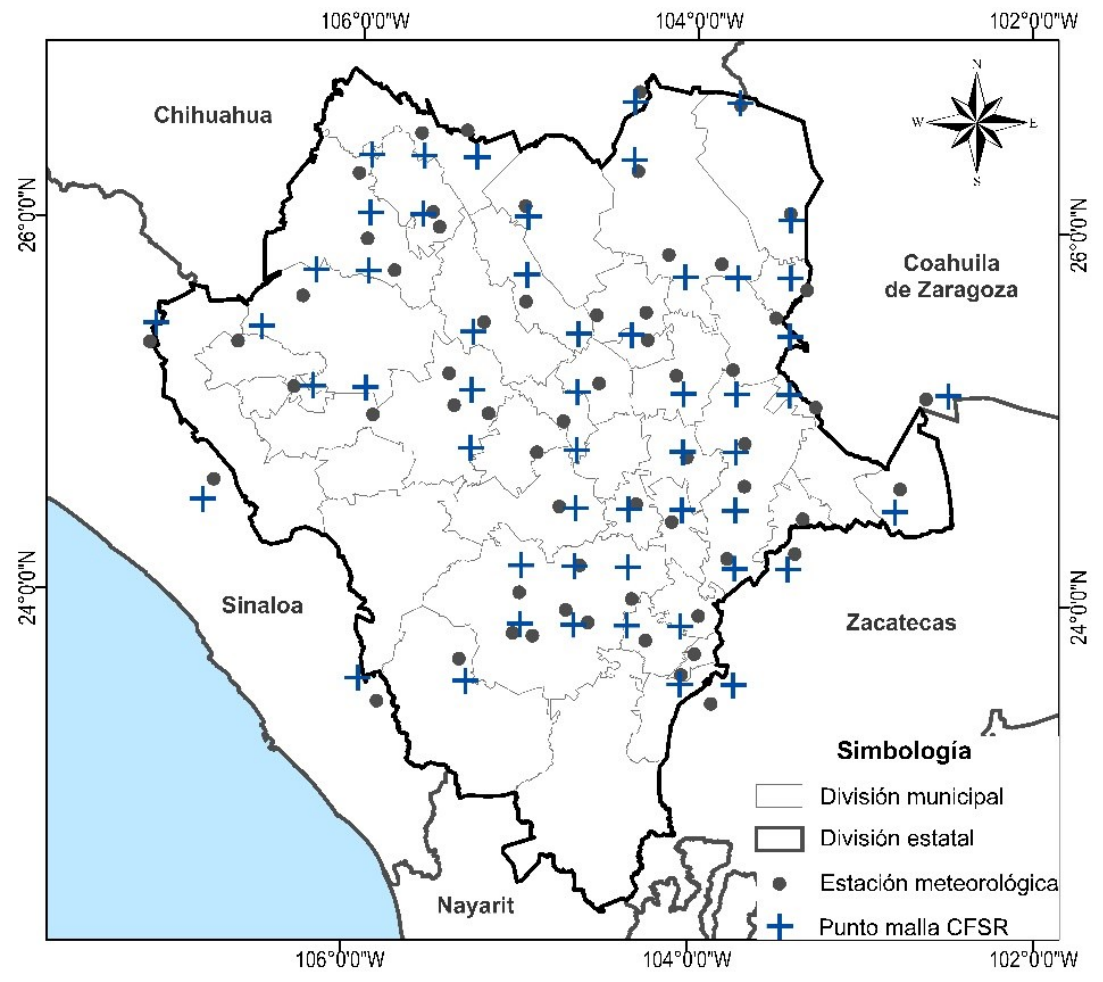

Figura 4. Puntos de la malla CFSR seleccionados.

Se completaron los datos faltantes en 59 estaciones con el procedimiento para cada variable y el método más adecuado. Para lo cual se evaluó la capacidad explicativa de los modelos sobre los datos 
2020, Instituto Mexicano de Tecnología del Agua

Open Access bajo la licencia CC BY-NC-SA 4.0

(https://creativecommons.org/licenses/by-nc-sa/4.0/)

medidos en las estaciones meteorológicas, a través de cincos índices estadísticos: cuadrado medio del error (MSE) (Sánchez-Algarra, Barraza-Sánchez, Reverter-Comas, \& Vegas-Lozano, 2006); raíz cuadrada del cuadrado medio del error (RMSE) (Lehmann \& Casella, 1998); error absoluto medio (MAE) (Fuertes \& Javier, 1998); índice de Willmott $(d)$ (Willmott, 1982), y el coeficiente de determinación $\left(R^{2}\right)$ (Infante \& Zarate, 2012).

Cada índice tiene un criterio para determinar si el modelo es un buen estimador; de esta forma se eligió como mejor método para estimar datos faltantes aquel en el que al menos tres índices estadísticos lo señalaran como el mejor estimador.

El modelo CFSR para estimar temperatura máxima en 34 estaciones resultó ser el mejor estimador de datos perdidos en comparación con las 25 que se completaron con el método NWS. El método NWS es el mejor estimador para precipitación, pues se evaluó como mejor en 57 estaciones, mientras que el modelo CFSR fue el mejor sólo en dos. Para temperatura mínima, el método NWS se evaluó como mejor en 41 estaciones. De esta manera se obtuvieron 61 series climáticas mensuales completas para las variables temperatura máxima y mínima, y precipitación. 
Tecnología y

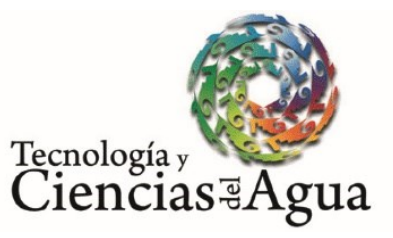

2020, Instituto Mexicano de Tecnología del Agua

Open Access bajo la licencia CC BY-NC-SA 4.0

(https://creativecommons.org/licenses/by-nc-sa/4.0/)

\section{Metodología}

\section{Índices de sequía}

Se utilizaron los índices de sequía Índice de Precipitación Estandarizada (SPI) y el Índice de Precipitación y Evapotranspiración Estandarizado (SPEI) para el análisis de la sequía. De acuerdo con las recomendaciones de la Organización Meteorológica Mundial (OMM, 2016), ambos ya han sido probados en el área de estudio (Rivera, Crespo, Arteaga, \& Quevedo, 2007; Llanes et al., 2018) y se pueden aplicar a partir de la información disponible.

El SPI se desarrolló por Mckee, Doesken y Kleist (1993), con el objetivo de cuantificar los impactos del déficit de precipitación sobre los diferentes recursos hidrológicos: agua subterránea, agua almacenada, corrientes de agua y humedad del suelo. El índice se define con base en la precipitación estandarizada, que es la diferencia de precipitación de la media para un periodo de tiempo específico dividido por la desviación estándar, donde la media y la desviación estándar se determinan a partir de registros pasados. 
Se calcula con base en un conjunto de datos mensuales de precipitación, el cual se está moviendo en el sentido de que cada mes se determina un nuevo valor a partir de los meses anteriores. Cada uno de los conjuntos de datos se ajusta a la función Gamma, para definir la relación entre probabilidad y precipitación. Dado que la función Gamma en análisis climáticos es su integral, se obtienen probabilidades de ocurrencia de una precipitación menor o igual que una precipitación determinada. Con el propósito de facilitar la obtención del índice SPI, la probabilidad acumulada se transforma a una variable normalizada $Z$ (con media cero y varianza uno), que representa el valor de SPI.

EI SPEI fue desarrollado por Vicente, Beguería y López (2010). Es un índice de sequía multiescalar, que combina datos de precipitación y temperatura. Funciona a partir del procedimiento original de cálculo del SPI. EI SPEI utiliza la diferencia mensual (o semanal) entre la precipitación y evapotranspiración (PET). Esto representa un balance hídrico climático simple que se fija en diferentes escalas de tiempo para obtener el SPEI.

El cálculo de PET resulta difícil por la cantidad de parámetros que intervienen en el proceso. El objetivo de incluir PET en el cálculo del índice de sequía es obtener una estimación temporal relativa; por lo tanto, el método que se utiliza para calcular el PET no es crítico, es decir, que SPEI no se encuentra vinculado con ningún método en particular. 
Para calcular SPEI se necesita una distribución de tres parámetros, ya que puede tener valores negativos, que son comunes en la serie de balance entre precipitación y evapotranspiración. Vicente et al. (2010) seleccionaron la función de densidad de probabilidad Log-logística, con base en el comportamiento de los valores más extremos. Después es transformada a una distribución normal estándar con media 0 y varianza 1. Dado que SPEI es una variable estandarizada, se puede comparar con otros valores de SPEI a lo largo del tiempo y el espacio.

Mckee et al. (1993) utilizaron un sistema de clasificación para definir intensidades de sequía (Tabla 1) y definieron criterios para cuando ocurre un evento de sequía, para cualquier escala de tiempo. Ya que SPEI es una variable estandarizada, al igual que SPI, se utiliza el mismo sistema de clasificación de sequías para ambos índices.

Tabla 1. Clasificación de SPI y SPEI.

\begin{tabular}{|c|c|}
\hline Valor del índice & Categoría del índice \\
\hline 2 o mayor & Extremadamente húmedo \\
\hline 1.5 a 1.99 & Muy húmedo \\
1.0 a 1.49 & Moderadamente húmedo \\
\hline-0.99 a 0.99 & Cercano a lo normal \\
\hline-1.0 a -1.49 & Moderadamente seco \\
\hline-1.5 a -1.99 & Muy seco \\
\hline
\end{tabular}




\begin{tabular}{|c|c|}
\hline Valor del índice & Categoría del índice \\
\hline-2 o menor & Extremadamente seco \\
\hline
\end{tabular}

Se calcularon los índices SPEI y SPI para las series climáticas con el programa RStudio ${ }^{\circledR}$ (RStudio Inc., 2018), con la paquetería SPEI (Begueria, Serrano, \& Sawasawa, 2013). Se obtuvieron los índices SPEI y SPI a escalas de 6 y 12 meses; además de la información climática de temperatura máxima y mínima, y precipitación, se utilizó la latitud de cada estación. Se seleccionó el método de Hargreaves para estimar la evapotranspiración.

\section{Comprobación de normalidad}

El método de Rodionov utiliza la prueba $t$-student como parte del procedimiento en la detección de cambios. Para emplear el procedimiento de la prueba $t$ se debe cumplir el supuesto de que los datos proceden de una distribución normal (Montgomery, 2010). 
Para comparar la normalidad de los datos se eligió la prueba de bondad, la prueba de Kolmogorov-Smirnov modificada por Lilliefors (1967). Se aplicó la prueba a cada serie de índices de sequía; se realizó mediante la paquetería nortest de RStudio $($; se utilizó un nivel de significancia (alfa, a) de 0.01 .

\section{Método de Rodionov para detectar cambios de régimen}

Rodionov (2004) propuso un método secuencial para detectar cambios de régimen (en la media y desviación estándar) como solución al problema de las series temporales de no consideran las datos finales de los métodos existentes para la detección de cambio de régimen y que en consecuencia se detectan mucho después de su aparición real.

El método consiste en realizar una prueba para determinar la validez de la hipótesis nula $H_{0}$ (en este caso, la existencia de un cambio de régimen). Hay tres posibles resultados de la prueba: aceptar $H_{0}$, rechazar $H_{0}$ o seguir realizando la prueba. 
2020, Instituto Mexicano de Tecnología del Agua

Open Access bajo la licencia CC BY-NC-SA 4.0

(https://creativecommons.org/licenses/by-nc-sa/4.0/)

En una serie de tiempo, cuando llega una nueva observación - que lo hace de forma regular-, se hace una comprobación para determinar si representa una desviación estadísticamente significativa del valor medio del régimen actual. Si es el caso, ese año se marca como un posible punto de cambio $c$, y las observaciones posteriores se utilizan para confirmar o rechazar esta hipótesis (Rodionov \& Overland, 2005).

La hipótesis se prueba al usar el índice de cambio de régimen (RSI), que se calcula para cada $c$ :

$R S I_{c}=\sum_{i=c}^{c+m} \frac{x_{i}{ }^{*}}{l \sigma_{l}}$

Donde $m=0, \ldots, l-1$ es el número de años desde el inicio de un nuevo régimen; $l$, la longitud de corte de los regímenes a probar, y $\sigma_{l}$ es la desviación estándar promedio para todos los intervalos de un año en las series de tiempo. RSI representa una suma acumulativa de desviaciones normalizadas $x_{i}{ }^{*}$ del nivel medio hipotético para el nuevo régimen $\left(\bar{x}_{n v o}\right)$, para el cual la diferencia del nivel medio para el régimen actual $\left(\bar{x}_{a c t}\right)$ es estadísticamente significativa de acuerdo con una prueba $t$ de Student. 
2020, Instituto Mexicano de Tecnología del Agua

Open Access bajo la licencia CC BY-NC-SA 4.0

(https://creativecommons.org/licenses/by-nc-sa/4.0/)

dif $=\bar{x}_{n v o}-\bar{x}_{a c t}=t \sqrt{2 \sigma_{l}^{2} / l}$

Donde $t$ es el valor de la distribución $t$-student con $2 /-2$ grados de libertad a un nivel de probabilidad $p$ dado. Si en cualquier momento desde el comienzo del nuevo régimen el RSI se vuelve negativo, la prueba falla y se asigna un valor cero. Si el RSI permanece positivo durante $l-1$, entonces $c$ se explica como el tiempo de un cambio de régimen en el nivel $\leq p$. La búsqueda del próximo cambio de régimen comienza con $c+1$ para garantizar que su sincronización se detecte de forma correcta, incluso si la duración real del nuevo régimen es $<1$ año.

El valor promedio del régimen actual $\bar{x}_{a c t}$ se calcula para el periodo $(c-l, c)$. Se calcula para el periodo desde el cambio de régimen anterior al punto inmediatamente anterior al punto actual en el tiempo. Como resultado, se produce una función gradual de los regímenes en casi todos los casos y no sólo para cambios abruptos en el régimen.

Los cambios en la varianza de alguna variable meteorológica pueden tener un impacto similar o incluso mayor en los ecosistemas que las variables en la media. Como los cambios climáticos debidos a causas naturales o humanas pueden ser en la frecuencia de los eventos 
2020, Instituto Mexicano de Tecnología del Agua

Open Access bajo la licencia CC BY-NC-SA 4.0

(https://creativecommons.org/licenses/by-nc-sa/4.0/)

extremos, representan el peligro de favorecer el aumento del "calentamiento global" (Rodionov, 2005b).

Rodionov desarrolló una extensión para Excel (Rodionov, 2005b) que detecta cambios en la varianza, procedimiento similar al de la media, excepto que es con base en la prueba $F$ en lugar de la prueba $t$.

Los datos de entrada para el programa son los valores de los índices de sequía y los parámetros de la prueba: longitud de corte, nivel de significancia y función de peso de Huber.

Los parámetros de longitud de corte y nivel de significancia controlan la magnitud y escala de los regímenes que fueron detectados. Diferentes combinaciones en estos parámetros tienen un efecto directo sobre el valor de RSI, es decir, se obtendrán diferentes interpretaciones de los resultados (Rodionov, 2006a). De esta manera, se analizó el caso de una longitud de corte igual a cinco para un nivel de significancia de 0.05 .

El parámetro de peso de Huber controla la participación de los valores atípicos. Debido a estos valores, el promedio no es representativo del valor medio de los regímenes. El peso para el valor de los datos se debe elegir de modo que sea pequeño si ese valor se considera como un valor atípico (Rodionov, 2006a). En función de la desviación estándar se usó un peso de Huber de 2. 
Tecnología y

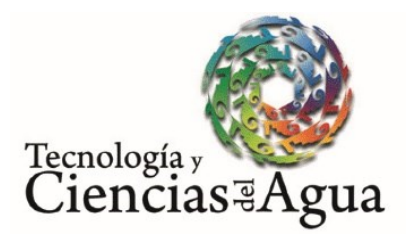

2020, Instituto Mexicano de Tecnología del Agua

Open Access bajo la licencia CC BY-NC-SA 4.0

(https://creativecommons.org/licenses/by-nc-sa/4.0/)

\section{Análisis de tendencias de cambios en media y varianza de los índices de sequía}

Para cada estación se calcularon las frecuencias de ocurrencia de cambios en la media (RSI) y varianza (RSSI) de los índices de sequía; para los valores RSI se calculó sólo la frecuencia de los valores RSI negativos, que representan los regímenes de sequía.

Se hizo un análisis espacial de las frecuencias obtenidas de los valores RSI y RSSI a través de la interpolación, que fue con base en el principio de autocorrelación y determina si hay un patrón espacial (Childs, 2004). La interpolación se hizo con la extensión Spatial Analyst de ArcGIS $®$ (ESRI, 2016), con el método kriging (Villatoro, Henríquez, \& Sancho, 2008).

Para el análisis de tendencias, las frecuencias de ocurrencia de cambios en la media y varianza se agruparon en periodos de 5 y 10 años. Se identificó si durante estos periodos se presentó una tendencia de disminución o incremento del número de cambios. 
Tecnología y

2020, Instituto Mexicano de Tecnología del Agua

Open Access bajo la licencia CC BY-NC-SA 4.0

(https://creativecommons.org/licenses/by-nc-sa/4.0/)

\section{Resultados}

Se calcularon los índices de sequía SPEI y SPI de seis meses para el periodo de junio de 1979 a diciembre de 2008; para los índices SPEI y SPI de seis meses, los valores obtenidos son de diciembre 1979 a diciembre de 2008. En la Tabla 2 se presentan las estadísticas de los índices de sequía.

Tabla 2. Estadísticas generales de los índices de sequía para el estado de Durango, México.

\begin{tabular}{|c|c|c|c|c|c|}
\hline Índice de sequía & Media & $\begin{array}{c}\text { Desviación } \\
\text { estándar }\end{array}$ & Mínimo & Máximo & Rango \\
\hline SPI de 6 meses & 0.009 & 0.984 & -3.025 & 2.46 & 5.485 \\
\hline SPI de 12 meses & 0.003 & 0.986 & -2.752 & 2.376 & 5.128 \\
\hline SPEI de 6 meses & 0.008 & 0.979 & -2.308 & 2.268 & 4.576 \\
\hline SPEI de 12 meses & 0.006 & 0.978 & -2.157 & 2.161 & 4.318 \\
\hline
\end{tabular}


2020, Instituto Mexicano de Tecnología del Agua

Open Access bajo la licencia CC BY-NC-SA 4.0

(https://creativecommons.org/licenses/by-nc-sa/4.0/)

La media de los índices (SPI y SPEI) es muy cercana a cero, lo cual indica que los valores extremos (periodos de sequías y de humedad) ocurren con la misma frecuencia. Es decir, que la presencia de las sequías durante el periodo de estudio es una característica normal del clima de la región, resultado similar a lo que presentó Contreras (2005).

En términos de la magnitud de los promedios de valores extremos, es mayor para los valores mínimos en tres de los índices analizados (SPI de 6 y 12 meses, y SPEI de seis meses). Esto indica que la magnitud de la sequía es mayor con respecto a la magnitud de los periodos húmedos. Ello se traduce en que las sequías representan mayor riesgo en el área cuando se presentan.

De las pruebas de normalidad, se cumplieron en 46 estaciones para el índice SPI de seis meses; 45, para SPEI de seis meses; 32, del índice SPI de 12 meses, y 31 para SPEI de 12 meses. Se realizó la prueba de Rodionov para la detección de cambios de régimen para los índices SPI y SPEI de seis meses, pues son los que cuentan con un mayor número de estaciones que cumplen la condición de normalidad, esto, con la finalidad de incluir una mayor superficie en el análisis. 


\section{Análisis del cambio de régimen en la media (RSI) y la varianza (RSSI)}

Para la media de los índices SPI y SPEI de seis meses se obtuvo el RSI; la media de los regímenes con pesos iguales; el valor medio de los regímenes calculados con la función de ponderación de Huber; la longitud de los regímenes; el nivel de confianza final para los cambios, y el peso (en función de la desviación estándar) de los valores atípicos.

Para la varianza, se obtuvo el índice de cambio de régimen de la varianza promedio (RSSI) y, al igual que para la media, se obtuvo la longitud del régimen y el nivel de confianza para los cambios.

Con los valores de RSI y RSSI se calcularon las frecuencias de ocurrencia de los valores de cambio por estación para el periodo 19792008.

Para RSI se obtuvieron las frecuencias de los valores negativos, que son los que indican el cambio hacia un régimen de sequía. Para cada estación se obtuvieron los valores mínimos y máximos de RSI y RSSI, respectivamente, así como la fecha en que se presentaron.

Los valores mínimos promedio de RSI son -3.23 para el índice SPEI de seis meses y -2.96 para SPI de seis meses. El promedio de 
2020, Instituto Mexicano de Tecnología del Agua

Open Access bajo la licencia CC BY-NC-SA 4.0

(https://creativecommons.org/licenses/by-nc-sa/4.0/)

valores máximos de RSSI es de 1.30 para SPEI de seis meses y 2.00 para SPI de seis meses.

Para los cambios en la media se tiene mayor frecuencia de ocurrencia de valores máximos en una fecha específica; mientras que para los cambios en la varianza, la frecuencia es más constante.

A través del análisis espacial se identificaron áreas del estado que muestran mayor frecuencia de cambios de régimen en la media y varianza. Para el RSI, en ambos índices, los valores de menor ocurrencia se presentan al sur del estado, que comprende un área mayor en el índice SPI. Una estación que se ubica en el noroeste presentó la mayor frecuencia para el índice SPEI; mientras que para el índice SPI, la frecuencia de cambios RSI fue la menor (Figura 5). 


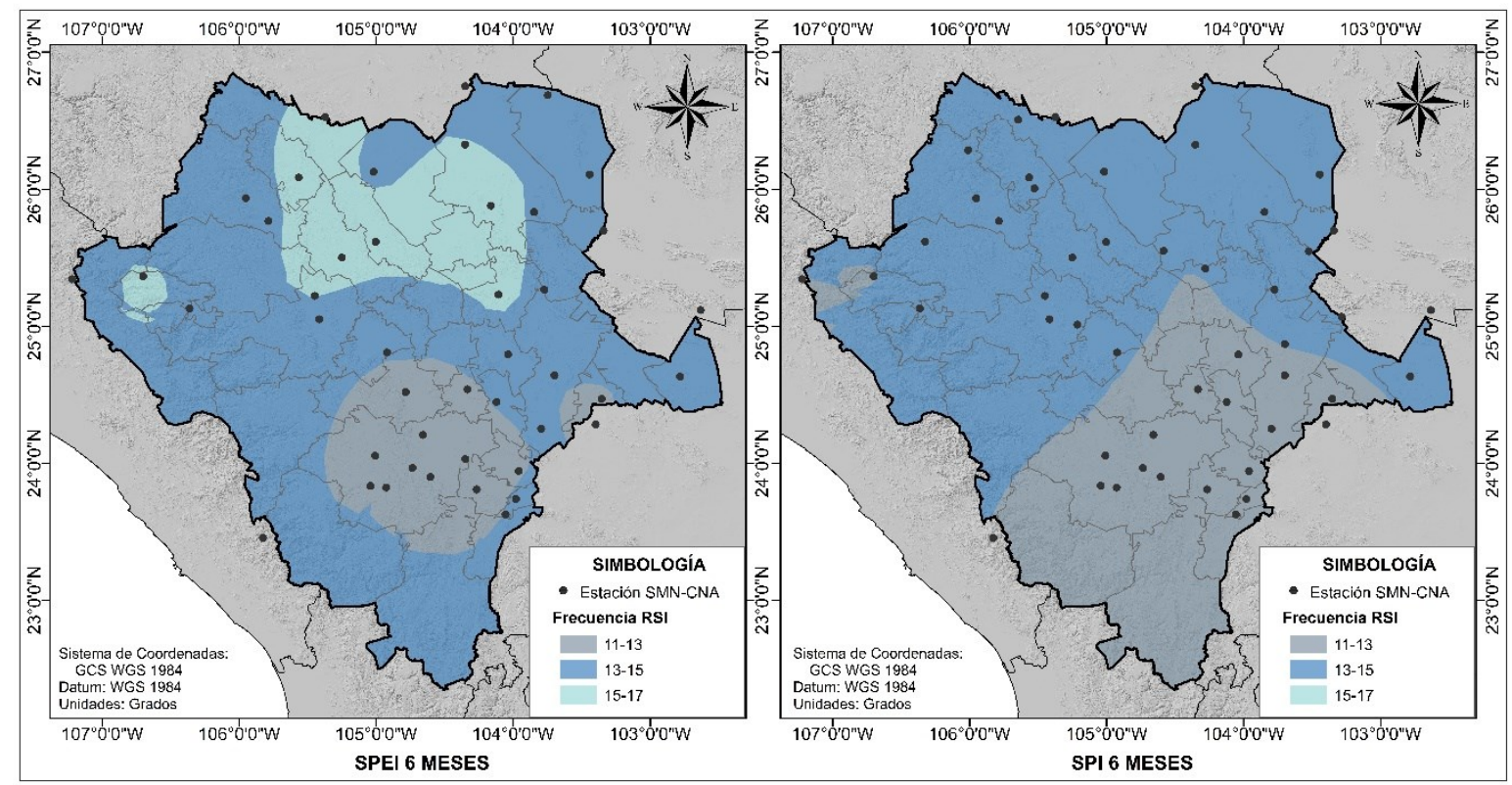

Figura 5. Distribución espacial de la frecuencia de valores RSI durante el periodo 1979-2008 en el estado de Durango México.

En la Figura 6 se observa que la frecuencia de los valores de cambio en la varianza RSSI va de 5 a 13 durante el periodo 1979-2008. Para el índice SPEI de seis meses, la menor frecuencia se presenta al oeste del estado, a modo de una franja que va de norte a sur, excluyendo una pequeña porción en los límites con Chihuahua y Sinaloa, que presenta una frecuencia de 7 a 9 ocurrencias, al igual que la mayor parte del estado, ya que las frecuencias mayores sólo representan una pequeña área al este, correspondiente con valores de sólo dos estaciones climatológicas. 
Tecnología y

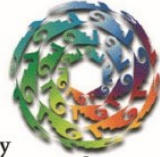

Ciencias : : Agua
2020, Instituto Mexicano de Tecnología del Agua

Open Access bajo la licencia CC BY-NC-SA 4.0

(https://creativecommons.org/licenses/by-nc-sa/4.0/)

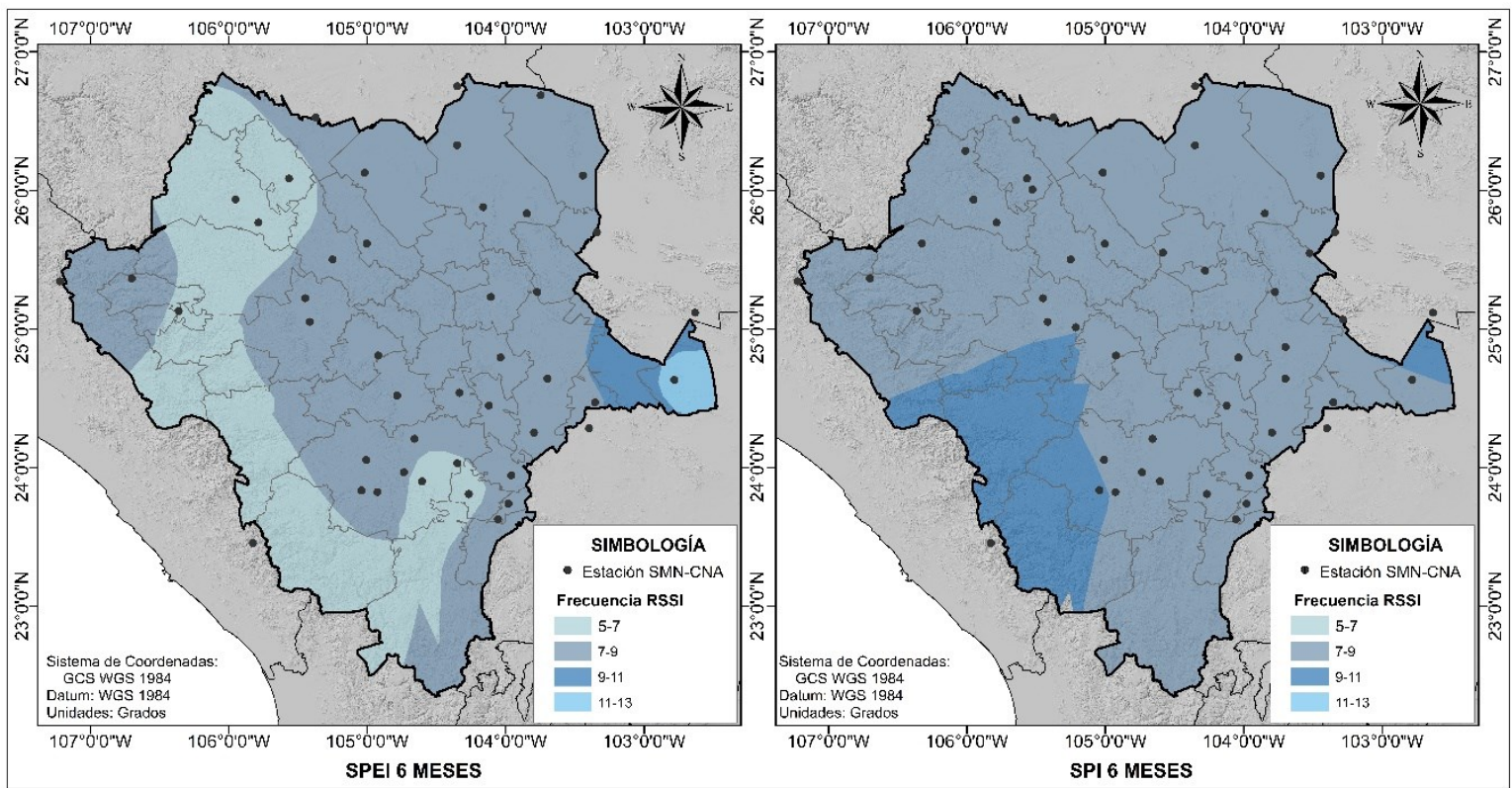

Figura 6. Distribución espacial de la frecuencia de valores RSSI durante el periodo 1979-2008 en el estado de Durango, México.

La frecuencia de RSSI para el índice SPI de seis meses presentó dos categorías de ocurrencia de 7 a 9 que cubre la mayor parte del estado; de 9 a 11 distribuida al oeste (resultado de dos estaciones), y una pequeña porción al este (de una estación).

La detección de cambios en la media y varianza depende del índice de sequía utilizado. Al emplear el índice SPEI, las frecuencias muestran mayor variabilidad, al agruparse en mayor número de categorías que las frecuencias obtenidas para el índice SPI. 
2020, Instituto Mexicano de Tecnología del Agua

Open Access bajo la licencia CC BY-NC-SA 4.0

(https://creativecommons.org/licenses/by-nc-sa/4.0/)

De acuerdo con el análisis espacial, la región norte del estado presenta mayor frecuencia de cambios; la estación que muestra mayor número de cambios es la 10078 Sardinas.

Por lo cual, a continuación se presenta un análisis individual de los resultados obtenidos en esta estación para los valores de RSI y RSSI (cambios en media y varianza de los índices de sequía, respectivamente).

Se representa en la gráficas (Figura 7) los valores de los índices de sequía, así como los valores de RSI y RSSI. Para los valores de RSI negativos, se ubicaron en el inicio de los periodos de sequía para los índices SPEI y SPI de seis meses, es decir que, el método detectó todos los cambios hacia un régimen de sequía. 

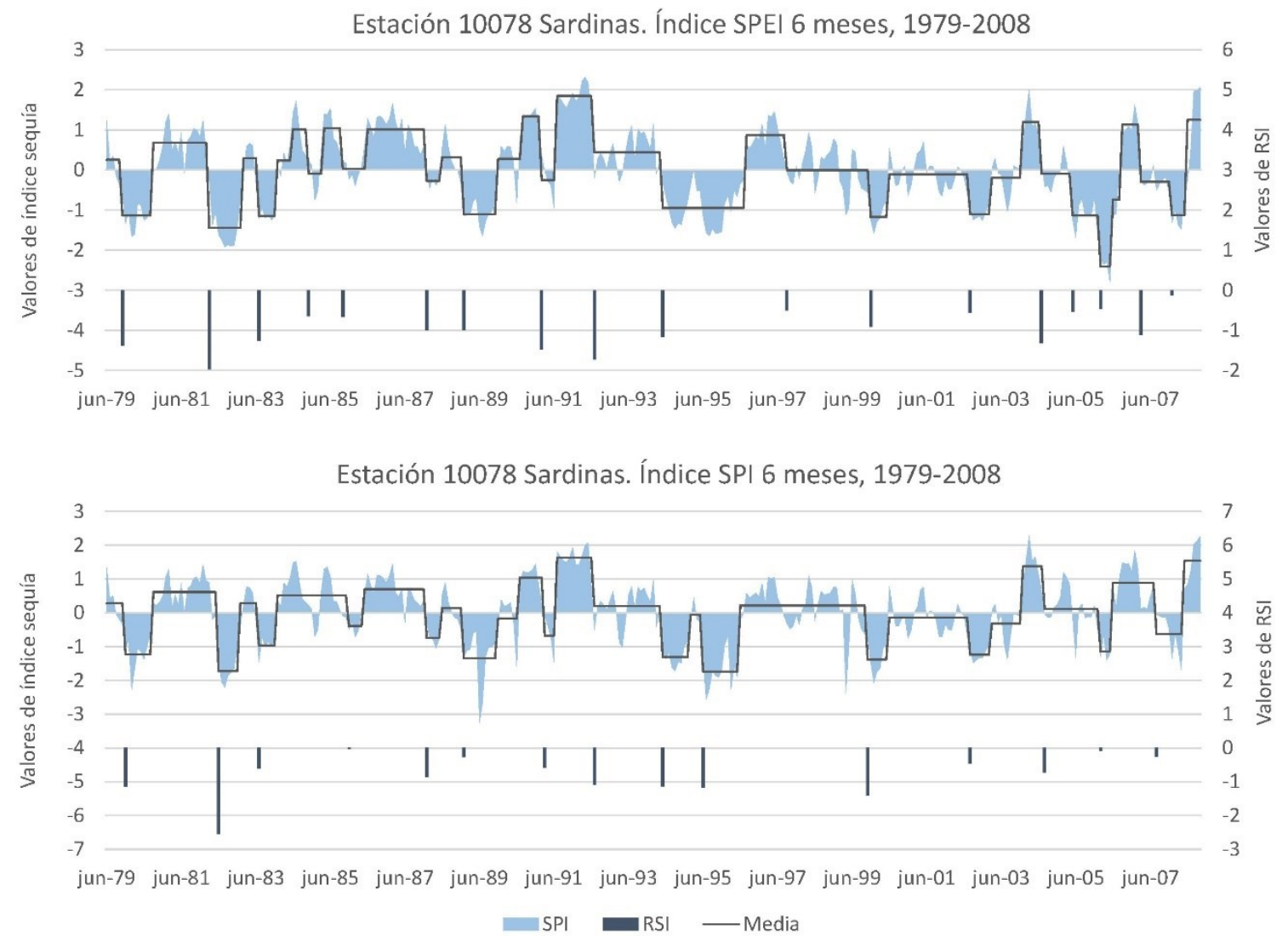

Figura 7. Valores de cambio RSI para los índices SPI y SPEI de seis meses en la estación10078 Sardinas.

Hubo 18 números de cambios en la media para el índice SPEI de seis meses, 18 en total; y 16 cambios para SPI en el mismo periodo. La mayor parte de los valores RSI se calculó en la misma fecha para ambos índices. La detección de cambios en la media depende entonces del 
índice de sequía con el cual se trabaje (SPEI o SPI), siendo más sensible con el índice SPEI.

De 1994 a 1996 se presentó el mayor periodo de sequía en la estación 10078 Sardinas. En este periodo se observan dos cambios negativos en la media. El valor de RSI es útil para detectar si una sequía continuará, pues el valor negativo de RSI indica que los valores de clasificación de sequía de los índices seguirán en la misma tendencia.

Los valores mayores de RSI se tienen cuando el cambio de régimen es más abrupto; en cambio, cuando la media es más constante, el cambio se da de forma gradual; los valores de RSI son de menor magnitud. Si los índices de sequía se encuentran en valores grandes de humedad (positivo) o de sequía (negativo), el cambio a un valor grande de la condición contraria resulta en valores de RSI de mayor magnitud.

Los valores máximos de RSI ocurrieron en diversas fechas en las estaciones analizadas, sin embargo se detectó que una gran parte ocurrió durante los meses de junio y julio de 1992.

Los cambios de la varianza no indican un comportamiento general de la sequía, sino que es particular de acuerdo con el índice utilizado. Además, la varianza es sensible a los valores extremos, por lo que la interpretación de los resultados se inclina hacia un cambio rápido en los valores de los índices de sequía. 
Tecnología y

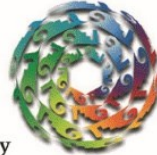

Ciencias
2020, Instituto Mexicano de Tecnología del Agua

Open Access bajo la licencia CC BY-NC-SA 4.0

(https://creativecommons.org/licenses/by-nc-sa/4.0/)

Los cambios detectados en la varianza para la estación 10078 Sardinas (Figura 8) indican una varianza media constante en prácticamente todo el periodo. Para SPEI de seis meses, los cambios en la varianza se ubican en los años 1985 a 1987; para SPI, la mayor parte de las variaciones se detectaron al final de la serie, de 2005 a 2007.

Estación 10078 Sardinas. Índice SPEI 6 meses, 1979-2008

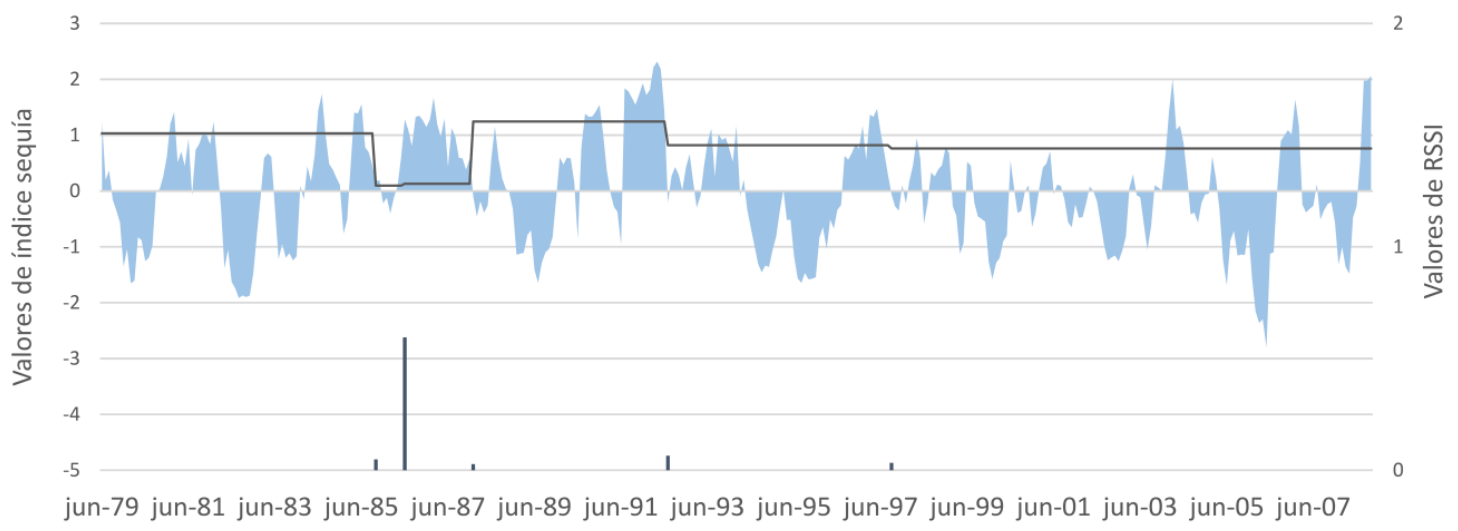

Estación 10078 Sardinas. Índice SPI 6 meses, 1979-2008

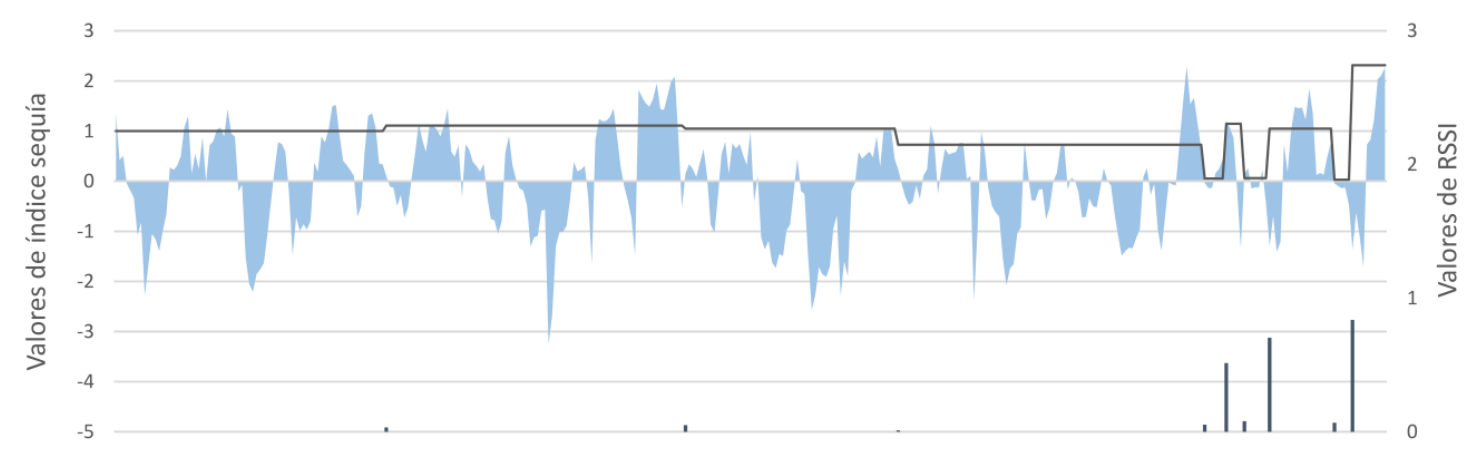

jun-79 jun-81 jun-83 jun-85 jun-87 jun-89 jun-91 jun-93 jun-95 jun-97 jun-99 jun-01 jun-03 jun-05 jun-07

$$
\text { SPI RSSI - Varianza }
$$


2020, Instituto Mexicano de Tecnología del Agua

Open Access bajo la licencia CC BY-NC-SA 4.0

(https://creativecommons.org/licenses/by-nc-sa/4.0/)

Figura 8. Valores de cambio RSSI para los índices SPI y SPEI de seis meses en la estación 10078 Sardinas.

En la estación 10078 Sardinas, los valores máximos de RSSI no coinciden con los valores máximos de RSI; en cambio, los máximos de RSSI se observan en una variación de la media de los índices de sequía, lo cual es de esperarse, ya que el índice de varianza indica la dispersión de los valores con respecto a su media.

Con lo anterior se puede inferir que los valores de RSSI serán útiles para detectar cambios hacia un periodo prolongado de sequía. Sin embargo, cuando los periodos de humedad se alternen con periodos de sequía con la misma magnitud y duración, no se detectarán cambios en la varianza. Por lo cual, los valores de RSSI se deben utilizar como un complemento de los valores RSI. Con ambas pruebas es posible que se detecten con mayor certeza los periodos de sequía prolongados y de mayor severidad y duración.

Se analizó la frecuencia de los valores de cambio RSI y RSSI de la media y varianza en los índices de sequía mediante la suma de valores cada 5 y 10 años. De esta manera, se identificó la tendencia de la frecuencia de cambios; es decir, si ha aumentado o disminuido la ocurrencia de cambios en la media y varianza de los índices de sequía en el tiempo. 
2020, Instituto Mexicano de Tecnología del Agua

Open Access bajo la licencia CC BY-NC-SA 4.0

(https://creativecommons.org/licenses/by-nc-sa/4.0/)

La tendencia de la frecuencia de cambios en la media cada cinco años (quinquenal) para los índices de sequía SPEI y SPI de seis meses se indica en la Figura 9. La frecuencia de ambos índices es parecida; sólo durante el periodo 1995-1999 la frecuencia de cambios para el índice SPEI de seis meses es menor que para SPI de seis meses. Durante los primeros cuatro quinquenios se observa una tendencia de aumento en los cambios en la media, y después durante el periodo 2000-2004 disminuye radicalmente; en el último periodo de 2004-2008, los cambios aumentan de nuevo hasta valores similares que en los primeros quinquenios.

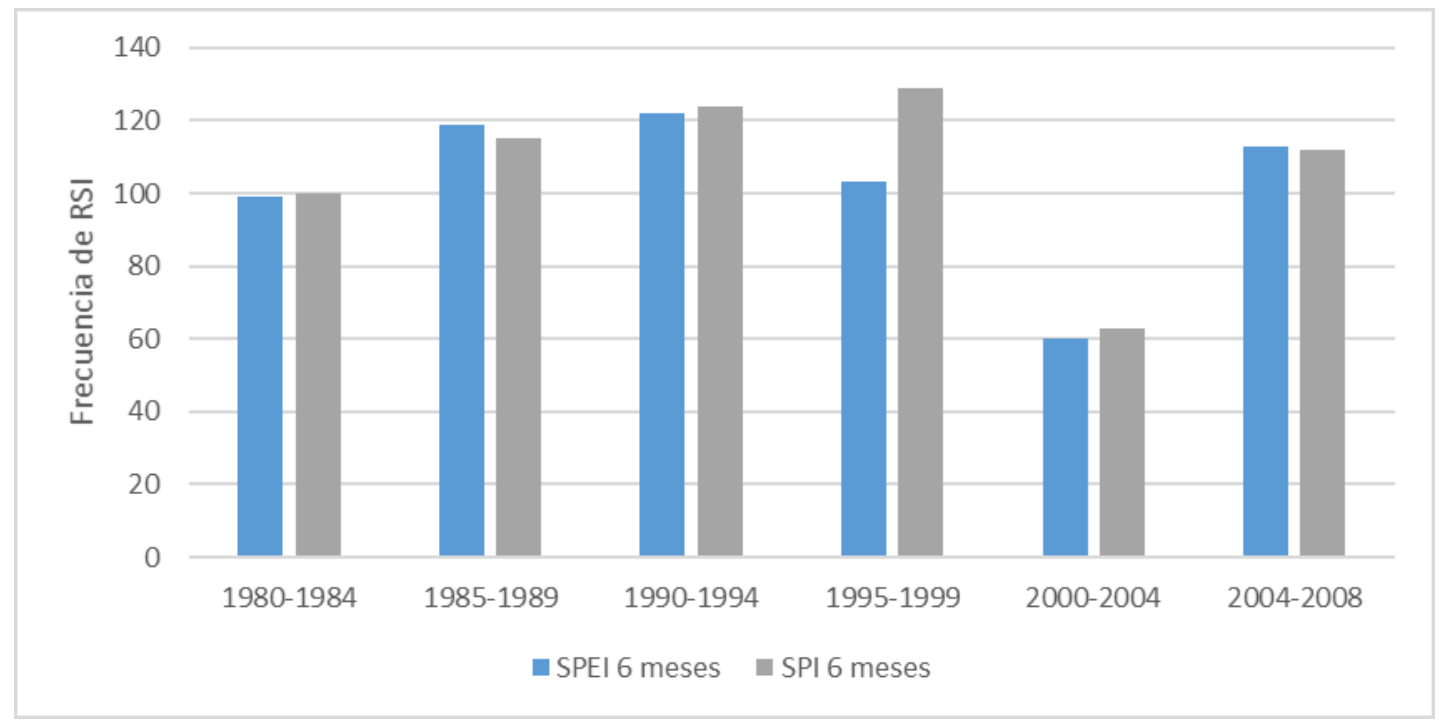

Figura 9. Frecuencia quinquenal de cambios en la media (RSI) durante el periodo 1980-2008. 
Tecnología y

Ciencias $₫$ Agua
2020, Instituto Mexicano de Tecnología del Agua

Open Access bajo la licencia CC BY-NC-SA 4.0

(https://creativecommons.org/licenses/by-nc-sa/4.0/)

La frecuencias de cambios en la media cada diez años (decadal) se tiene en la Figura 10. Se observa que durante la última década (20002008) ocurrió la menor cantidad de cambios, y durante el periodo 19901999 se presentó la mayor frecuencia de valores RSI.

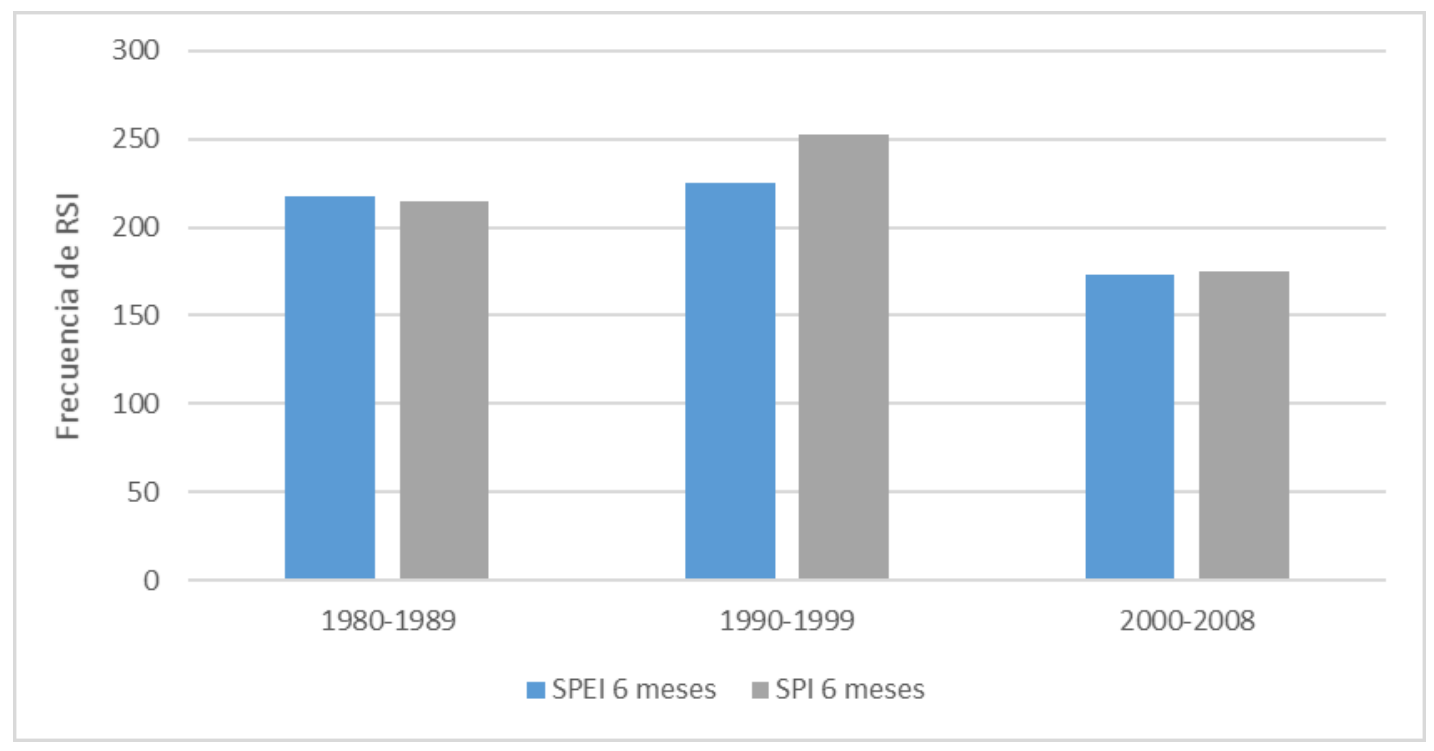

Figura 10. Frecuencia decadal de cambios en la media (RSI) durante el periodo 19802008.

Los resultados obtenidos coinciden con los encontrados por Llanes et al. (2018), donde se concluye que los eventos extremos de sequía se 
presentaron durante los años de 1990 y 1997, siendo los años intermedios un periodo de sequías recurrentes.

Para la frecuencia de cambios en la varianza, los índices de sequía SPI y SPEI de seis meses se muestran en la Figura 11, donde se observa una mayor frecuencia ( $>40$ ) de cambios para el índice SPI de seis meses en todos los periodos; la frecuencia de RSSI es mayor para el SPI con respecto a SPEI.

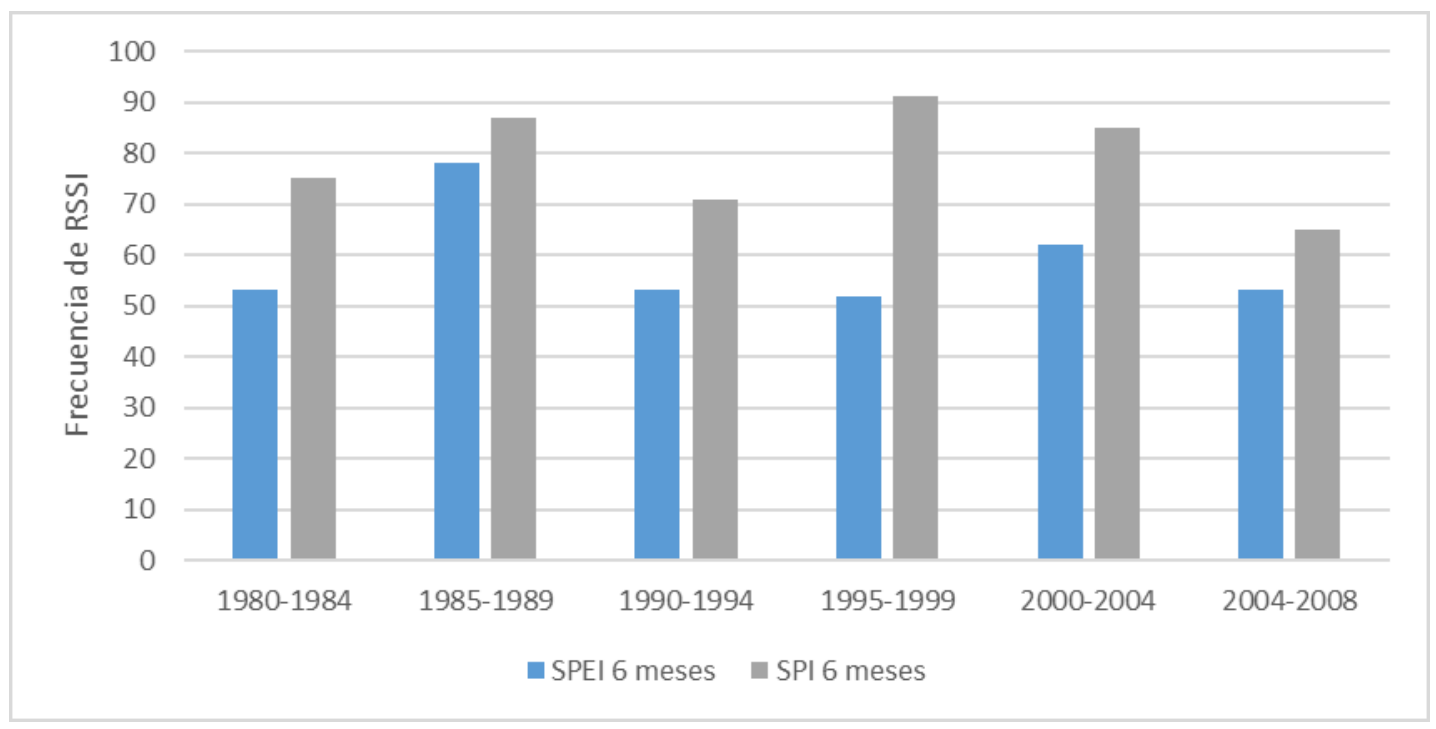

Figura 11. Frecuencia quinquenal de cambios en la varianza (RSSI) durante el periodo 1980-2008.

La frecuencia de cambios en la varianza para periodos de diez años se muestra en la Figura 12. Se observa que la primera década de 
análisis (1980-1989) es la que presentó mayor frecuencia de cambios; sin embargo, para SPI, el valor se repite en la siguiente década, por lo que durante 20 años el promedio de cambios fue relativamente constante.

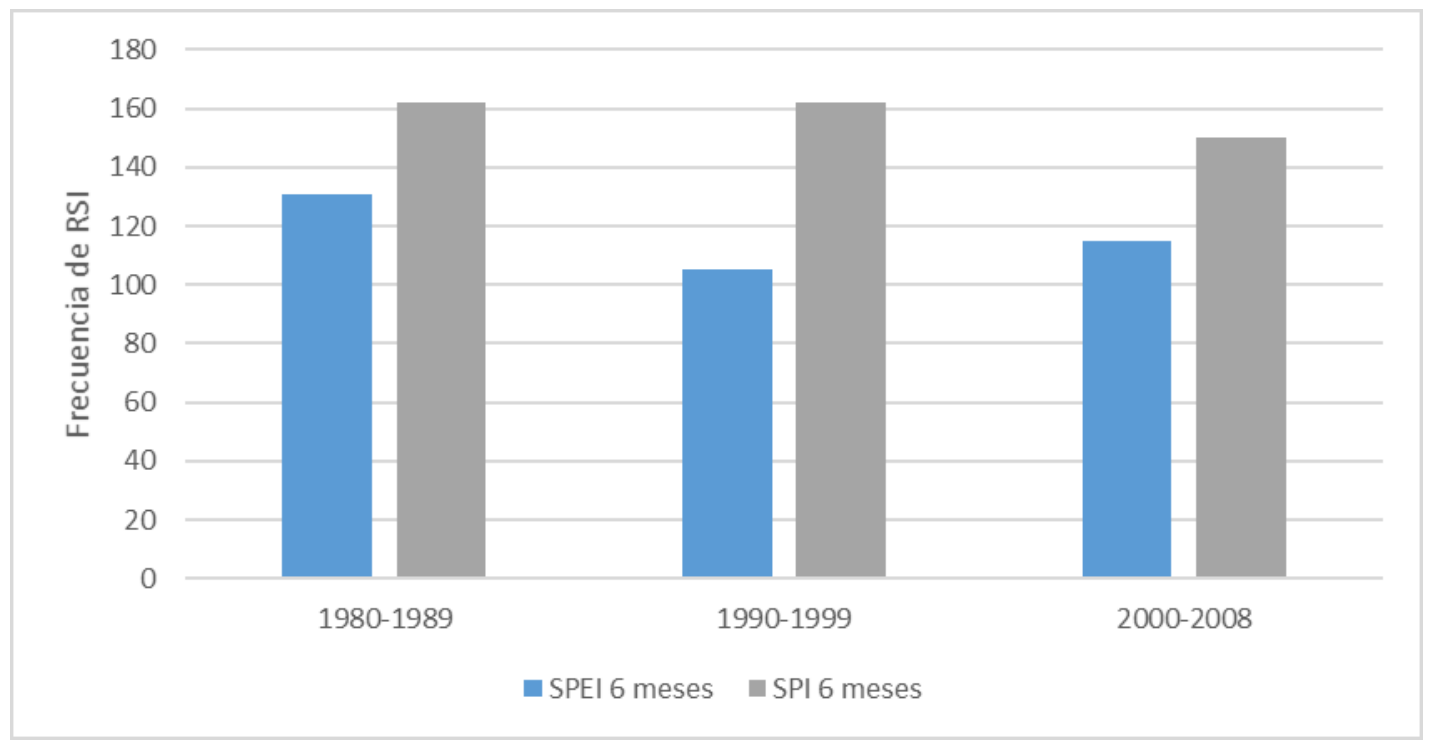

Figura 12. Frecuencia decadal de cambios en la varianza (RSSI) durante el periodo 1980-2008.

Los resultados obtenidos indican que los cambios en media y varianza no han sido mayores durante los últimos años del periodo analizado. 
Tecnología y

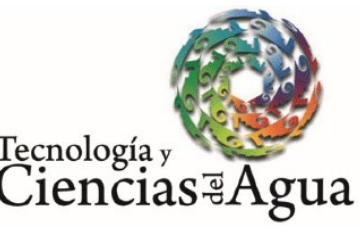

2020, Instituto Mexicano de Tecnología del Agua

Open Access bajo la licencia CC BY-NC-SA 4.0

(https://creativecommons.org/licenses/by-nc-sa/4.0/)

\section{Conclusiones}

Se analizó la sequía por medio de los índices de sequía SPI y SPEI a escalas temporales de 6 y 12 meses en el estado de Durango, México. Se identificaron los periodos de sequía con los valores de cambios de la media (RSI) de signo negativo. Se detectaron 14 cambios en promedio para el índice SPEI de seis meses y 13 cambios para el índice SPI de seis meses. Los valores de cambio en la varianza (RSSI) fueron ocho para el índice SPEI de seis meses y 10 para el índice SPI de seis meses.

Las tendencias de los cambios en la media y varianza de los índices de sequía con base en la frecuencia de los cambios en la media disminuyó durante el periodo 2000-2004 y aumentó durante 20052008. La década de mayores cambios en la media fue 1990-1999. Para la varianza se observó una frecuencia máxima de cambios durante el periodo 1985-1989; los cambios se mantuvieron constantes durante los últimos 20 años. La década de mayores cambios en la varianza fue de 1980-1989.

\section{Referencias}


Bates, B. C., Kundzewicz, Z. W., Wu, S., \& Palutikof, J. P. (2008). Climate change and water. Climate Change and Water. DOI:10.1016/j.jmb.2010.08.039

Begueria, S., Serrano, V., \& Sawasawa, H. (2013). SPEI: Calculation of Standardised Precipitation-Evapotranspiration Index. $R$ package version 1.6. DOI:10.1175/2009JCLI2909.1.http

Campos, D. F. (1998). Procesos del ciclo hidrológico. San Luis Potosí, México: Universidad Autónoma de San Luis Potosí.

Cancelliere, A., \& Salas, J. D. (2010). Drought probabilities and return period for annual streamflows series. Journal of Hydrology, 391(12), 77-89. DOI:10.1016/j.jhydrol.2010.07.008

Cenapred, Centro Nacional de Prevención de Desastres. (2002). Fascículo sequías. México, DF, México: Secretaría de Gobernación. Recuperado de http://www.cenapred.gob.mx/es/Publicaciones/archivos/8FASCCULOSEQUAS.PDF

Childs, C. (2004). Interpolating surfaces in ArcGIS spatial analyst. Education Service. ArcUser July-September. 32-34 p. Recuperado de http://www.esri.com

Contreras, C. S. (2005). Las sequías en México durante el siglo XIX. Investigaciones Geográficas, (56), 118-133. Recuperado de http://www.scielo.org.mx/pdf/igeo/n56/n56a8.pdf 
Domínguez, J. (2016). Revisión histórica de las sequías en México: de la explicación divina a la incorporación de la ciencia. Tecnología y ciencias del agua, 7(5), 77-93.

ESRI, Environmental Systems Research Institute. (2016). ArcMap I ArcGIS Desktop 10.5. Versión 10.5.0.6491. USA: Environmental Systems Research Institute, Inc.

Fuertes, S. J., \& Javier, S. (1998). Evaluación de dos métodos de determinación de la evapotranspiración de referencia en condiciones semiáridas. Recuperado de http://hdl.handle.net/10261/16644

Gallegos, J., Arteaga, R., Vázquez, M. A., \& Juárez, J. (2016). Estimation of missing daily precipitation and maximum and minimum temperature records in San Luis Potosí. Ingeniería Agrícola $y$ Biosistemas, 8(1), 3-16. DOI: 10.5154/r.inagbi.2015.11.008

Gamboa, R. O. (2015). Evaluación de modelos empíricos, matemáticos y redes neuronales para estimar datos faltantes en estaciones meteorológicas en México (tesis de maestría en ciencias), Postgrado de Hidrociencia, Colegio de Postgraduados.

Heim, R. R. (2002). A review of twentieth-century drought indices used in the United States. Bulletin of the American Meteorological 
Society, 83(8), 1149-1165. DOI: 10.1175/15200477(2002)083<1149:AROTDI >2.3.CO;2

Hounam, C. E., Burgos, J. J., Kalik, M. S., Palmer, W. C., \& Rodda, J. (1975). Drought and agriculture (Technical Note No. 138). World Meteorological Organization, (392), 129.

Ibarrarán, M. E., \& Rodríguez, M. (2007). Estudio sobre economía del cambio climático en México. Recuperado de http://inecc.gob.mx/descargas/cclimatico/e2007h.pdf

INEGI, Instituto Nacional de Estadística y Geografía. (2017). Anuario estadístico y geográfico de Durango 2017. Recuperado de http://internet.contenidos.inegi.org.mx/contenidos/Productos/prod _serv/contenidos/espanol/bvinegi/productos/nueva_estruc/anuari os_2015/702825077174.pdf

Infante, S., \& Zarate, G. P. (2012). Métodos estadísticos. Un enfoque interdisciplinario ( $3 a$ ed.). Colegio de Postgraduados. España: Mundi-Prensa.

Lehmann, E. L. \& Casella, G. (1998). Theory of point estimation. Springer Texts in Statistics ( $2^{\text {nd }}$ ed.) New York, USA: SpringerVerlag Inc.

Lilliefors, H. W. (1967). On the Kolmogorov-Smirnov Test for Normality with Mean and Variance Unknown. Journal of Statistical Association, 62(318), 399-402. DOI: 200.130.19.152 
Llanes, O., Gaxiola, A., Estrella, R. D., Norzagaray, M., Troyo, E., Pérez, E., Ruíz, R., \& Pellegrini, M. de J. (2018). Variability and factors of influence of extreme wet and dry events in Northern Mexico. Atmosphere, 9(4), 1-16. DOI: 10.3390/atmos9040122

Mckee, T. B., Doesken, N. J., \& Kleist, J. (1993). The relationship of drought frequency and duration to time scales. AMS 8th Conference on Applied Climatology, (January), 179-184. Recuperado de https://doi.org/citeulike-article-id:10490403

Miller, A. J., Cayan, D. R., \& Barnett, T. P. (1994). The 1976-77 climate shift of the Pacific Ocean. Oceanography, 7(1), 21-26.

Montgomery, D. C. (2010). Diseño y análisis de experimentos (2a ed.). México, DF, México: Limusa Wiley.

OMM, Organización Meteorológica Mundial. (2016). Manual de indicadores e índices de sequía. Programa de gestión integrada de sequías. Recuperado

de http://www.droughtmanagement.info/literature/WMOGWP_Manual-de-indicadores_2016

Ortega, D. (2013). Sequía: causas y efectos de un fenómeno global. Ciencia y Sociedad, 16(61), 8-15. Recuperado de https://www.researchgate.net/publication/260163188 
Rivera, R., Crespo, G., Arteaga, R., \& Quevedo, A. (2007). Temporal and spatial behavior of drought in the state of Durango, Mexico. Terra Latinoamericana, 25(1), 383-392.

Rodionov, S. (2004). A sequential algorithm for testing climate regime shifts. Geophysical Research Letters, 31(9), 2-5. DOI: 10.1029/2004GL019448

Rodionov, S. (2005a). A brief overview of the regime shift detection methods. In: Velikova, V., \& Chipev, N. (eds.). Large-scale disturbances (regime shifts) and recovery in aquatic ecosystems: Challenges for management toward sustainability. UNESCOROSTE/BAS Workshop on Regime Shifts (pp. 17-24), 14-16 June 2005, Varna, Bulgaria.

Rodionov, S. (2005b). A sequential method for detecting regime shifts in the mean and variance. Joint Institute for the Study of the Atmosphere and Ocean. Seattle, USA: University of Washington. Recuperado de https://www.beringclimate.noaa.gov/regimes/rodionov_var.pdf

Rodionov, S. (2006a). Help with regime shift detection software. Recuperado de https://www.beringclimate.noaa.gov/regimes/help3.html

Rodionov, S. (2006b). Sequential regime shift detection. Recuperado de https://www.beringclimate.noaa.gov/regimes/ 
Tecnología y

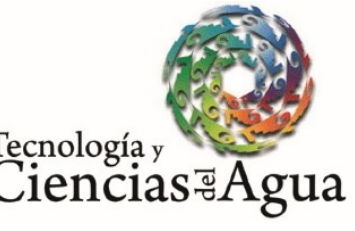

2020, Instituto Mexicano de Tecnología del Agua

Open Access bajo la licencia CC BY-NC-SA 4.0

(https://creativecommons.org/licenses/by-nc-sa/4.0/)

Rodionov, S., \& Overland, J. (2005). Application of a sequential regime shift detection method to the Bering Sea ecosystem. Journal of Marine Science, 62(May), 328-332. DOI: 10.1016/j.icesjms.2005.01.013

RStudio Inc. (2018). RStudio. Recuperado de https://www.rstudio.com/products/rstudio/download/

Saha, S., Moorthi, S., Pan, H.-L., Wu, X., Wang, J., Nadiga, S., Tripp, P., Kistler, R., Woollen, J., Behringer, D., Liu, H., Stokes, D., Grumbine, R., Gayno, G., Wang, J., Hou, Y. T., Chuang, H. Y., Juang, H. M. H., Sela, J., Iredell, M., Treadon, R., Kleist, D., Van Delst, P., Keyser, D., Derber, J., Ek, M., Meng, J., Wei, H., Yang, R., Lord, S., van den Dool, H., Kumar, A., Wang, W., Long, C., Chelliah, M., Xue, Y., Huang, B., Schemm, J. K., Ebisuzaki, W., Lin, R., Xie, P., Chen, M., Zhou, S., Higgins, W., Zou, C. Z., Liu, Q., Chen, Y., Han, Y., Cucurull, L., Reynolds, R. W., Rutledge, G., \& Goldberg, M. (2010). The NCEP Climate Forecast System Reanalysis. Bulletin of the American Meteorological Society, 91(8), 1015-1058. DOI:10.1175/2010BAMS3001.1

Saha, S., Moorthi, S., Wu, X., Wang, J., Nadiga, S., Tripp, P., Behringer, D., Hou, Y. T., Chuang, H. Y., Iredell, M., Ek, M., Meng, J., Yang, R., Peña-Mendez, M., van den Dool, H., Zhang, q., Wang, W., Chen, M., \& Becker, E. (2014). The NCEP Climate Forecast System 
Version 2. Journal of Climate, 27(6), 2185-2208. DOI: 10.1175/JCLI-D-12-00823.1

Sánchez-Algarra, P., Barraza-Sánchez, X., Reverter-Comas, F., \& VegasLozano, E. (2006). Métodos estadísticos aplicados. Barcelona, España: Universitat de Barcelona.

Semarnat, Secretaría de Medio Ambiente y Recursos Naturales. (2009). Programa Hídrico Visión 2030 del Estado de Durango. México, DF, México: Secretaría de Medio Ambiente y Recursos Naturales.

Vicente, S. M., Beguería, S., \& López, J. I. (2010). A multiscalar drought index sensitive to global warming: The standardized precipitation evapotranspiration index. Journal of Climate, 23(7), 1696-1718. DOI: $10.1175 / 2009 J C L I 2909.1$

Villatoro, M., Henríquez, C., \& Sancho, F. (2008). Comparación de los interpoladores idw y kriging en la variación espacial de ph, ca, cice y p del suelo. Agronomia Costarricence, 32(1), 95-105.

Willmott, C. J. (1982). Some comments on the evaluation of model performance. American Meteorological Society, 63(11), 13091313. DOI: $10.1175 / 1520-$

0477(1982)063<1309:SCOTEO>2.0.CO;2 